\title{
Fear, anxiety and their disorders: past, present and future neural theories
}

\author{
Neil McNaughton \\ University of Otago, Dunedin, New Zealand
}

\begin{abstract}
This paper reviews the historical development of a two-dimensional (direction x distance (?)) neural model of defense. It begins with Miller's (1944) analysis, and model, of approach, avoidance and conflict; adds Hinde's (1966) ethological perspective and Flynn's (1967) neural model of fear; and then considers Gray's (1967, 1970) work linking barbiturate action to the hippocampus, McNaughton's (1977) extension of this to other classes of anxiolytics, and Gray \& McNaughton's (1983) detailed behavioral comparison of anxiolytics and hippocampal lesions. This work led to Gray's (1982) detailed model of the neuropsychology of anxiety. Rapoport's (1989) model of the control of obsession by the cingulate cortex, and Ledoux's (1994) model of the control of both fear and anxiety to the amygdala, suggested a more complex organisation of defense systems. McNaughton (1989) argued that evolutionary function defines an emotion, and Blanchard and Blanchard (1990) argued for its assessment via ethoexperimental analysis. Graeff (1994) then produced a neural model that mapped defensive distance to neural level, treating all anxiety as being at a greater defensive distance than fear. Seeing this, and the treatment of anxiety as due to uncertainty (which is inconsistent with Miller's data), as being unsatisfactory, Gray and McNaughton (2000) and then McNaughton and Corr (2004) developed the two-dimensional model of defensive systems. This model is clearly incomplete at the present time and its links with neuroeconomics, personality, and stress and greater specification of frontal cortical contributions are suggested as directions for future development. Keywords: fear, anxiety, periaqueductal gray, hypothalamus, hippocampus, amygdala, cingulate cortex, frontal cortex
\end{abstract}

Received 4 February 2011; received in revised form 13 March 2011; accepted 17 March 2011. Available on line 10 October 2011

This paper describes the development of the twodimensional neural model of fear and anxiety (McNaughton $\&$ Corr, 2004). The ordering of topics in the paper is both close to the order in which I met them academically and the order of their occurrence historically - but I have made some departures from strict temporal sequence to make the development of parallel thought streams more coherent and to promote understanding of the content of the later theories.

"History" implies retrospection. But what I am telling is the story of how I and a field moved forward and, I look into the future because I can see the theory with which I finish being, for some current postgraduate student, the equivalent of Miller's theory of conflict, which started my own academic journey.

\section{Miller 1944: Conflict - The behaviorist perspective}

Approach and avoidance can seem to be opposite poles of a single dimension, requiring only a single system

Neil McNaughton, Dept. Psychology and Brain Health Research Centre, Department of Psychology, University of Otago, Dunedin, New Zealand. Correspondence regarding this article should be directed to Neil McNaughton,University of Otago. PO BOX 56, Dunedin, New Zealand. Telephone:+64-3-479-7643. Email: nmcn@psy.otago.ac.nz controlling movement in one direction or the other. Neal Miller (who shaped a number of Jeffrey Gray's early ideas) carried out a number of experiments on conflict leading to a two (approach/avoidance) system model. The key was to pit approach against avoidance by presenting both shock and food to a hungry rat in a straight alley. A range of experiments showed that distinct systems controlled approach and avoidance with, importantly, the strength of avoidance decreasing at a greater rate from the goal than that of approach (Figure 1).

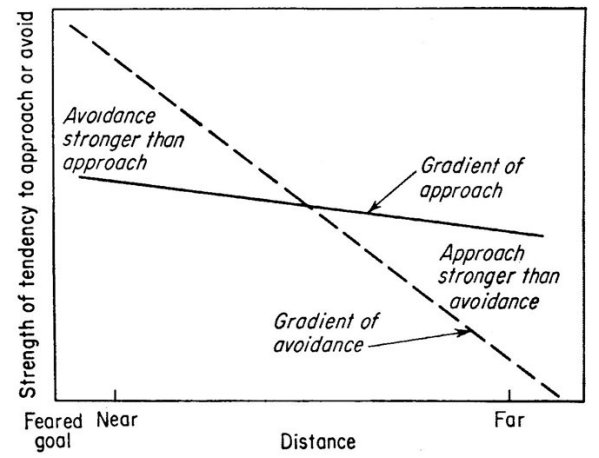

Figure 1. Miller's (1944) graphic summary of data on approach-avoidance conflict. "The tendency to approach is stronger far from the feared goal, while the tendency to avoid is stronger near the goal" taken with permission from Hinde (1966) p. 247. (C) The McGraw-Hill Companies Inc. 


\section{Miller model}

Miller developed a model (Figure 2) that explained much about the rate and direction of the rat's movement, including the dithering that tended to occur at the cross-over point of the two gradients. This was a fundamentally behaviorist model - focussing on approach and avoidance. But Miller viewed it also as a potentially neurally realistic model. I view him as the first in a line of fundamentally neural theorists because, among other things, he showed that the location of the conflict point, and the behavior that typically occurs at it, was sensitive to sodium amylobarbitone (an anxiolytic commonly prescribed by physicians at the time). Critically, the drug did not eliminate approach or avoidance but simply shifted the balance between them, providing early evidence of a distinct conflict system.

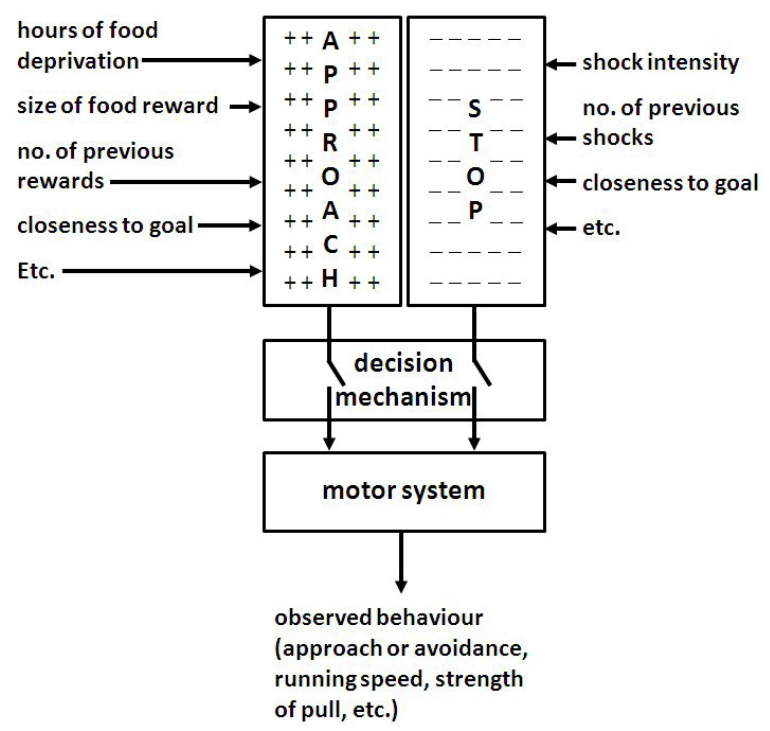

Figure 2. Miller's model, see text, redrawn from Gray (1971, p.120).

\section{Hinde 1966: Conflict-The ethological perspective}

In parallel with this behaviorist perspective, and linked to it, was analysis by ethologists. Thus, Figure 1 is a copy in Hinde's ethological text "Animal Behaviour" of Miller's original approach-avoidance analysis - but a key point, for Hinde, of the animal's behavior was that "...it may run a little way up, hesitate, turn back, and oscillate about a point some distance from the goal, or hesitate there grooming its fur or cleaning its paws" (Hinde, 1966, p. 247). That is, conflict is not just a state where approach and avoidance are balanced (which could explain dithering) but it is a state that generates other, relatively unexpected, "displacement" behaviors such as grooming (op. cit. pp. 278-9). This was another early indication of the operation of an additional system to those of pure approach and pure avoidance - one that could generate its own distinctive behavior as well as altering the balance between the other two.

\section{Flynn 1967: A fear system}

The early work was not explicit about the neural systems involved; nor about the way such systems could operate. In particular, it was not clear whether an "avoidance system" produced behavioral patterns that the experimenter could then label as fear-related, or whether the system generated a state that could be labelled "fear", which would then generate appropriate behavior.

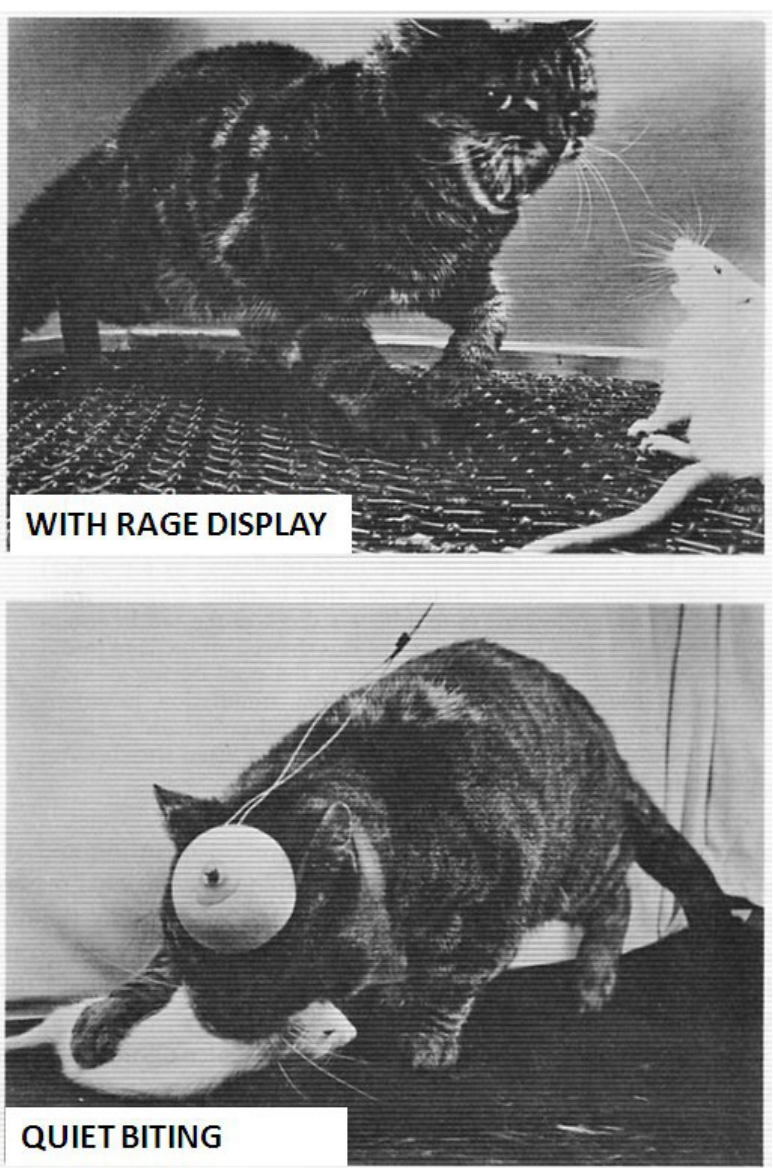

Figure 3. Above: Examples of two different forms of attack produced by stimulation of different systems within the hypothalamus. From Flynn (1967), p. 45, with permission. (C) Rockefeller University Press, 1967. Originally published in Neurophysiology and Emotion (D.C. Glass, editor).

This issue is addressed by experiments carried out by Flynn (1967) who found that stimulation of slightly different areas in the hypothalamus of a cat could cause it to attack a rat with two quite different attitudes (Figure 3). There was predatory attack involving quiet biting with no sign of fear or other aversive emotion from the cat; and there was a quite different form of attack (described by Flynn as accompanied by a display of rage), indicative of fear.

Particularly important points about these results are that, in both cases, if there was no stimulation there was no attack, and also if there was stimulation but no rat was present, there was no attack (although the rage display still occurred). Thus, the stimulation did not 
simply elicit the behavior, it created an internal state that produced a disposition to produce behavior - but the behavior that was produced was then shaped by environmental stimuli. Not only was a rat required to release the behavior but the behavior itself was shaped, on a moment by moment basis, by the specific behavior being shown by the rat. The stimulation, then, appeared to be eliciting the relevant emotional state.

The presumed fear state was elicited from a range of sites within the hypothalamus (Figure 4) suggesting that these were all part of a distinct hypothalamic fear system, separate from that controlling predation. This led Flynn to an explicit neural model of the release of behavior by the electrical stimulation that shared many elements with the ethological notions of release of displacement activities by conflict.

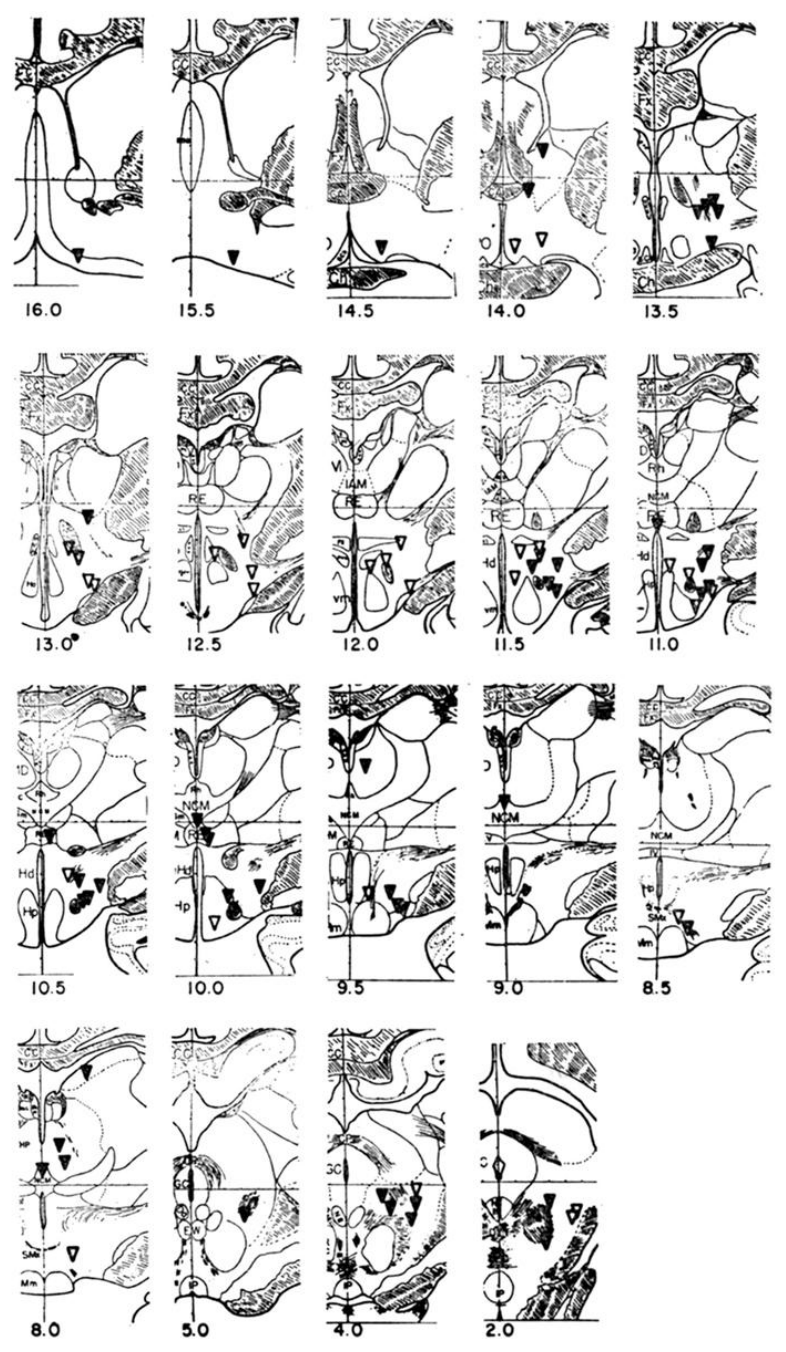

Figure 4. Electrode placements producing the two forms of display shown in Figure 3. Note that there appear to be two systems that run in parallel through the hypothalamus. Open triangles - attack with display. Filled triangles quiet biting. From Flynn (1967, p.55), with permission. (C) Rockefeller University Press, 1967. Originally published in Neurophysiology and Emotion (D.C. Glass, editor).

\section{Flynn model}

Flynn (1967) combined the hypothalamic stimulation data with a large number of other related experiments to produce a neurally explicit model (Figure 5). He distinguished between two types of motor output. $\mathrm{R}_{1}$ were essentially "elicited" behaviors (walking, sniffing, snarling, rage) that were produced by the stimulation in a largely unconditional manner. $\mathrm{R}_{2}$ were essentially "released" behaviors, particularly attack. The latter are controlled jointly by the patterning mechanism and external stimulus (S) input via a sensory filter and via a motor disposition. The effects of the patterning mechanism are arguably best seen as reflecting an emotional state that is set up when it is activated by the stimulation. Of course, under normal circumstances this emotional state would be created by other inputs (e.g., the facilitating mechanism) and also, in Flynn's model, could be inhibited.

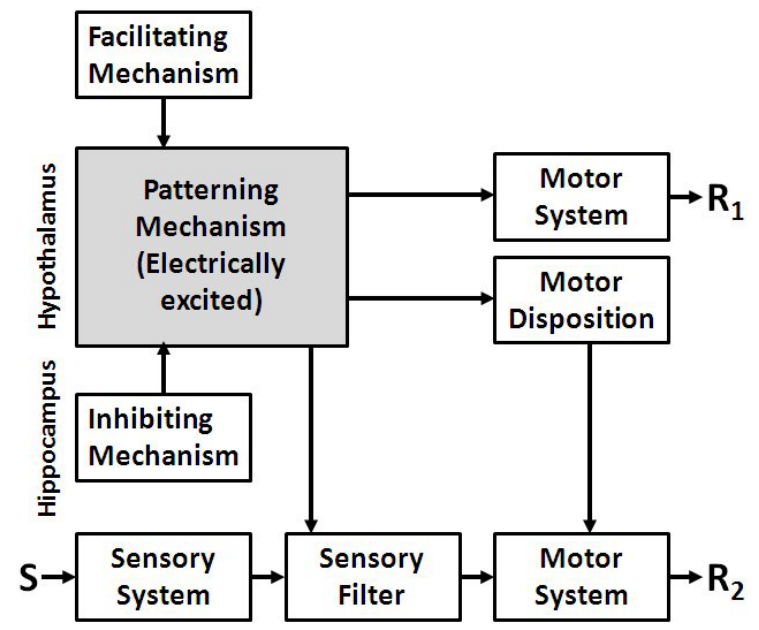

Figure 5. A model of the fear system to account for the data of Figures 3 and 4 and a large number of other experiments. For details, see text. $\mathrm{S}=$ external stimuli (the rat); $\mathrm{R}_{1}=$ "elicited" behaviors; $\mathrm{R}_{2}=$ "released" behaviors. Redrawn with modifications from Flynn (1967, p. 59).

A particularly valuable feature of Flynn's model is that, in his text, he explicitly specifies the neural loci for many of his mechanisms. The patterning mechanism is located in the hypothalamus; and at least part of the inhibiting mechanism is located in the hippocampus. This is one of the earliest models to identify the hippocampus with a behavioral inhibition system.

\section{Gray 1967, 1970 - Barbiturate as marker}

Following on to some extent from Miller's use of barbiturate drugs and taking a systems approach of the type advocated by Flynn, Gray used barbiturates as markers for systems that mediate anxiety. He concluded:

"there is a single physiological system which mediates the effects of both punishment and frustrative 
nonreward (the 'fear = frustration' hypothesis; Gray, 1967), and ... this system is antagonised by barbiturate drugs. ... It should be noted that the reduction of the effects of punishment ... is confined to passive avoidance situations... there is a remarkable similarity between the effects of lesions... to the hippocampus, and injections of amobarbital" (Gray, 1970, pp. 465466, my italics).

This statement is remarkable in that, at the time, it was based on just one drug and only a handful of behaviors (Gray, 1967). Yet it has shown strong subsequent behavioral and neural predictive validity.

\section{McNaughton 1977 - Theta frequency}

Observing a correlation and proving a cause is not the same thing. Gray went on to link the similar effects of amobarbital and hippocampal lesions on behavior to changes that the drug produced in hippocampal theta rhythm. The theta rhythm is a regular sinusoidal field potential that results from the rhythmic burst firing of hippocampal neurons. It is the main electrical pattern of activity in the hippocampus when a rat is performing voluntary movements (Vanderwolf, 1969). Septal driving of theta rhythm is affected by amylobarbitone (Gray \& Ball, 1970), but this effect failed to account for a substantial proportion of the similarity in behavioral effects of anxiolytics drugs and hippocampal lesions (McNaughton \& Mason, 1980). However, as part of my $\mathrm{PhD}$, I tested the effects of both amylobarbitone and a range of benzodiazepines and found (Figure 6) that anxiolytics (but not non-anxiolytic depressants) reduced the frequency of hippocampal theta rhythm

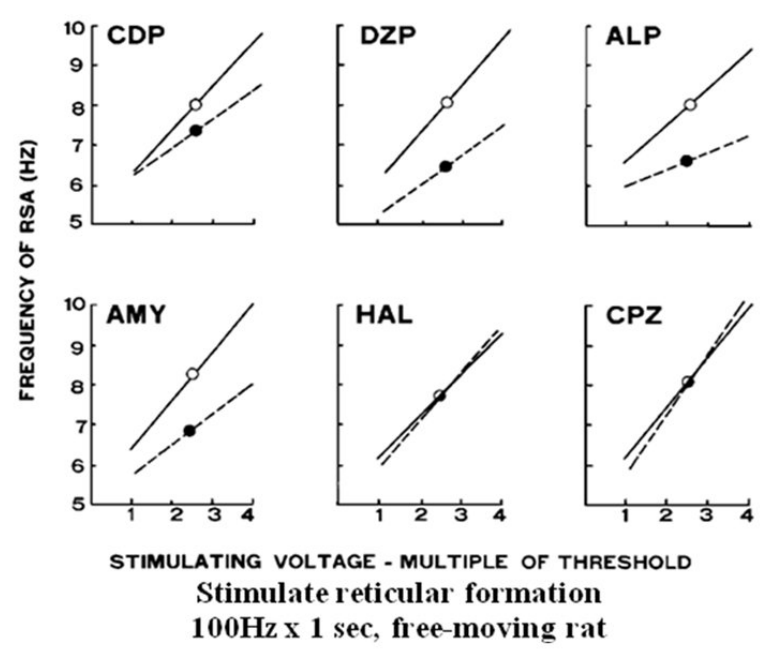

Figure 6. Classical anxiolytic drugs (barbiturates, benzodiazepines) reduce the frequency of hippocampal theta rhythm. Depressant drugs do not. From McNaughton and Sedgwick (1978). ALP = alprazolam; AMY = amylobarbitone; $\mathrm{CDP}=$ chlordiazepoxide; $\mathrm{CPZ}=$ chlorpromazine; $\mathrm{DZP}=$ diazepam; $\mathrm{HAL}=$ haloperidol.
(McNaughton \& Sedgwick, 1978). To skip forward in time, in terms of the historical sequence of this paper, my laboratory later showed that all drugs with an anxiolytic action (including ethanol, buspirone, imipramine, and fluoxetine) reduced theta frequency, whereas non-anxiolytics (anticholinergics, anti-serotonergics, anti-dopaminergics, anti-noradrenergics) did not (Coop \& McNaughton, 1991; Coop, McNaughton, \& Lambie, 1991; Coop, McNaughton, \& Scott, 1992; Coop, McNaughton, Warnock, \& Laverty, 1990; McNaughton \& Coop, 1991; Munn \& McNaughton, 2008; Zhu \& McNaughton, 1991a, 1991b, 1994, 1995). Currently, this test of anxiolytic action has no false positives, no false negatives, and has demonstrated predictive validity over three new drug classes (McNaughton, Kocsis, \& Hajós, 2007, for review).

This and later work allows the common behavioral profile of anxiolytics and hippocampal lesions to be explained by the fact that the drugs impair theta modulated processing in the hippocampal system.

\section{Gray \& McNaughton 1983 - Hippocampal profile}

The link between anxiolytic drug action and the hippocampus, and a much more detailed picture of the nature of the core common behavioral deficits, was provided by a detailed review of the profile of hippocampal lesion effects (Gray \& McNaughton, 1983). This provided a major foundation for Gray's model of the neuropsychology of anxiety (see next section) when combined with his previous review of the effects of anxiolytic drugs (Gray, 1977). This was further extended by Gray and McNaughton (2000), where (Table 1) the predictive validity of the original theory was demonstrated by the extension of the original profile from classical (GABA-acting) anxiolytic drugs to novel (serotonergic) ones, and to the inclusion (for both classes of drug) of effects on the classic test of hippocampal spatial processing, the Morris water maze. The most important feature of the table was the retention of the rule, originally noted in relation to amylobarbitone, that the drugs and hippocampal lesions change passive-avoidance-like behavior but not activeavoidance-like behavior.

\section{Gray 1982 model-The neuropsychology of anxiety}

The main elements of Gray's (1982) model of the processing of anxiety are shown in Figure 7. The key point of this model, in relation to later models, is that the hippocampal formation (HF) is essentially seen as a single core 'comparator' structure that processes information related to anxiety. Fear processes are located elsewhere, and the bottom left-hand part of the 
Table 1. Comparison of the behavioral effects of hippocampal lesions (HIP) and classical and novel anxiolytic drugs (ANX). $0=$ no effect; $+=$ behavior increased/performance improved; - = behavior decreased/performance impaired. Adapted from Gray and McNaughton (2000)

\begin{tabular}{lcc}
\hline Task & HIP & ANX \\
\hline Rewarded bar pressing, CRF & 0 & 0 \\
Rewarded running, CRF & 0 & 0 \\
Simultaneous discrimination & 0 & 0 \\
Shock, skilled escape, active avoidance & 0 & 0 \\
Resistance to extinction & + & + \\
Two-way active avoidance & + & + \\
Non-spatial active avoidance & + & + \\
Rewarded bar pressing, intermittent RF & + & + \\
Successive discrimination & - & - \\
Spontaneous alternation & - & - \\
Rearing & - & - \\
Mazes (not radial arm) & - & - \\
Passive avoidance & - & - \\
Differential reinforcement of low rates & - & - \\
Spatial discrimination reversal & - & - \\
Water maze & - & - \\
\hline & - & - \\
\hline
\end{tabular}

model incorporates the main elements of Flynn's model, particularly the inhibitory function of the hippocampus. Behavioral inhibition of flexible behavior (as opposed to fixed action patterns) occurs via the Papez circuit.

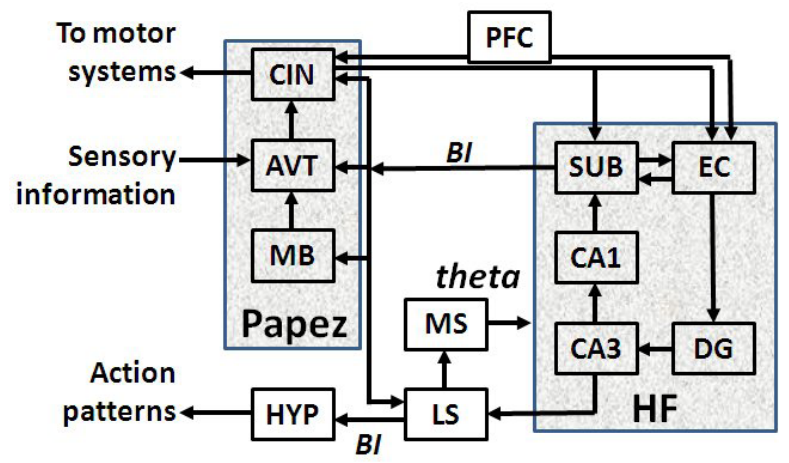

Figure 7. Gray's (1982) model of the anxiety system (simplified from his original diagram). The hippocampal formation (HF) processes information related to anxiety. The theta rhythmic input to the hippocampus is altered by anxiolytic drugs and this is how they alter the main functional outputs, particularly behavioral inhibition $(\boldsymbol{B I})$, see text. HYP = hypothalamus, c.f. Figure 5 .

\section{Rapoport 1989 - Obsession}

Gray's model is essentially unitary - attempting to keep anxiety and fear each confined to a single place, or at least to a homogenous system, in the brain. However, even at the time, Gray recognised that there was a form of anxiolytic-insensitive anxiety (or possibly fear) characterised by obsessionality and likely controlled via frontal cortical areas. Rapoport (1989) provided an even more separate view of obsession. A cross-over design (Figure 8) showed that clomipramine was clearly more effective than not only anxiolytics but also tricyclic antipanic drugs at treating obsession. This suggests that obsession is a distinct function from simple anxiety, or simple fear (at least as indexed by panic), with its own distinct neurology.

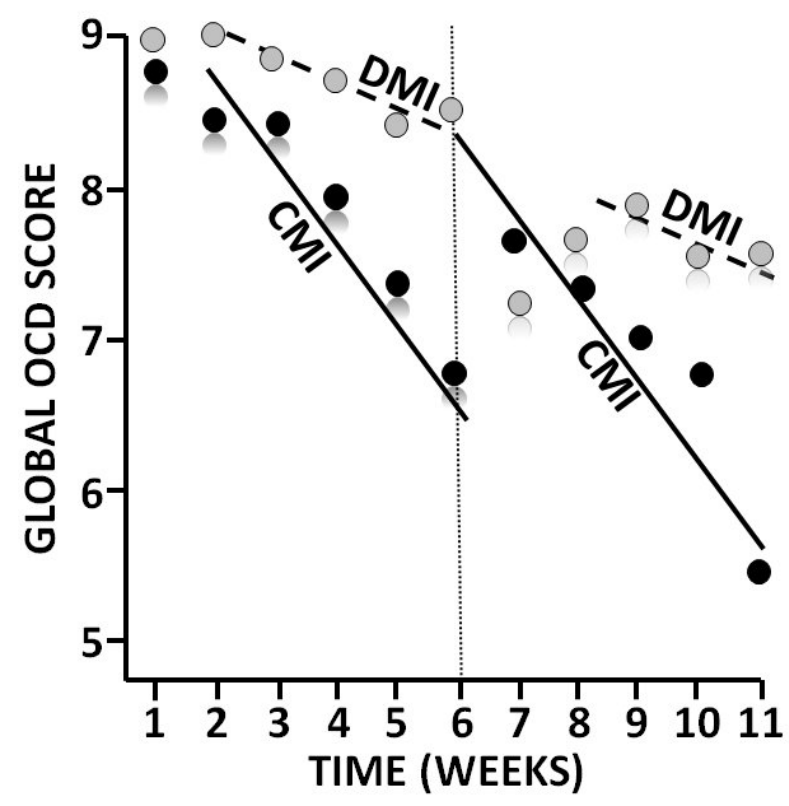

Figure 8. Results of a drug cross-over experiment comparing the effect of clomipramine (CMI, black circles) with desipramine (DMI, gray circles) in the treatment of obsession. Left-hand side: the group treated with CMI shows clear reductions in obsessionality as measured by a clinician-assessed global OCD score. Right-hand side: the group switched to DMI from CMI shows an increase in scores that returns them to values that would be expected with continuous DMI. The group switched to CMI from DMI shows a reduction in scores similar to that of the original CMI group. With each pair of lines (solid, dashed) the slopes are the same. Adapted from Rapoport (1989).

Rapoport (1989) provided considerable evidence to link the generation of obsessions with the cingulate cortex and the generation of compulsions with the caudate nucleus. This not only makes it appear distinct from simple fear and simple anxiety but also links it to a range of disorders involving repetitive behavior. 


\section{Ledoux 1994 - Fear/Anxiety Model}

A further complication for Gray's (1982) model was the development of theories of the amygdala that linked it to both fear and anxiety. One particularly influential model is that of LeDoux (LeDoux, 1994). According to his view, the role of the hippocampus was as a cognitive processor, dealing particularly with contextual stimuli, whereas the main control of both fear and anxiety was located, according to LeDoux, within the amygdala (Figure 9). This model both appears to ignore the extensive data discussed above linking anxiolytic action with the hippocampus and appears to locate two quite distinct functional systems within a single structure. It also places the hippocampus at the top of a progressively more complex hierarchy of processors. This seems unlikely in terms of phylogenetic development with the hippocampus being relatively older and showing much less expansion than the neocortex.

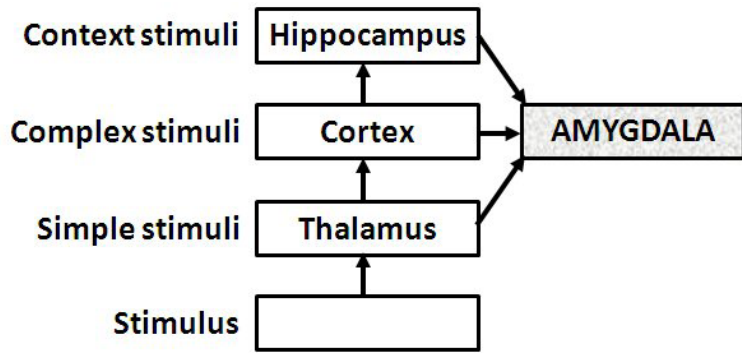

Figure 9. Ledoux's model of the control of fear and anxiety by the amygdala. The hippocampus is treated simply as a processor of highly complex, contextual, stimuli. Adapted from LeDoux (1994).

\section{McNaughton 1989 - Evolutionary function as defining an emotion}

The idea of phylogeny brings us to a second strand of theoretical development that was progressing in parallel with the neuropsychological analyses. To maintain the coherency of this report, I deal with this strand by taking a small step backward in time to my analysis of the possible value of biological, and particularly evolutionary, considerations in solving the age-old problem of "what is an emotion" (James, 1884). My conclusion (McNaughton, 1989) was that emotions are best seen as being only partially coherent at the neural, or control systems, level. The best means of characterising an emotion, I argued, was via its teleonomy (Pittendrigh, 1958), which can be treated as an evolutionary function in a retrospective or historical sense. That is, each emotion includes multiple reactions that evolved separately without the need for a common neural control, but are perceived as coherent because they have evolved for a common "purpose" (or it is better to say that the selection of the random mutations giving rise to them was shaped by the same, recurring, fundamental situational requirements).

\section{Blanchard \& Blanchard 1990 - Ethoexperimental analysis}

These conclusions, then, naturally drew me to the work of Robert and Caroline Blanchard. They argued strongly that typical controlled laboratory experiments fail to make clear why behavior occurs and that this is a significant problem for theoretical development.

"An additional and very different approach to [emotion] stems from analysis of [the] possible functional significance [of] characteristic behavior patterns seen in response to important ecological demands (e.g., feeding, reproduction, defense) when animals are given the rather wide range of behavioral choices typical of most natural habitats, is called ethoexperimental analysis. It involves a view that the functional significance of behavior attributed to anxiety (or other emotions) needs to be taken into account; ... [which] can be determined far more efficiently when the behavior is studied under conditions typical of life for the particular species". (Blanchard \& Blanchard, 1990, p.125)

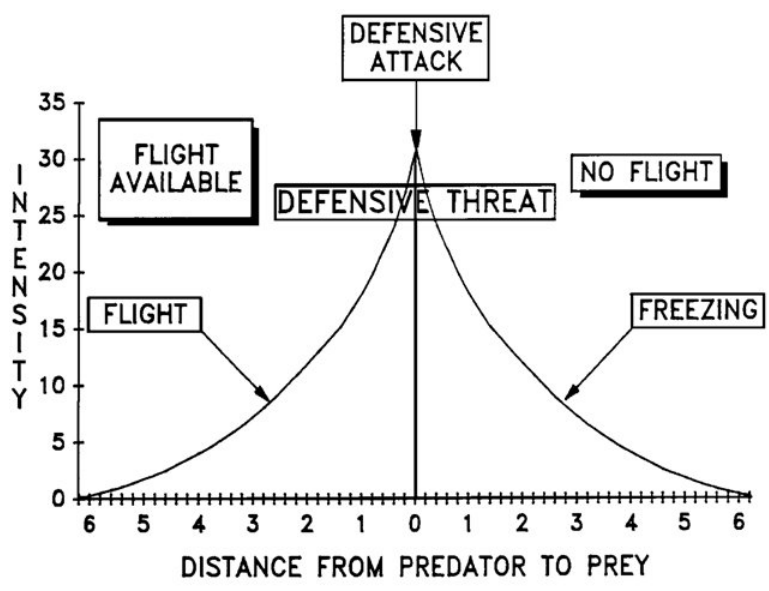

Figure 10. Schematic representation of the relation of behavior to defensive distance. From Blanchard and Blanchard (1990), with permission of the authors.

They, therefore, carried out experiments using a "visible burrow system" that allowed detailed analysis of the normal day to day behavior of rats and of its interference by the introduction of a cat. For me, they had two particularly important findings. First, large amounts of behavioral variation could be understood in terms of a hierarchy controlled by "defensive distance" (Figure 10). Specific behaviors occurred at specific "distances" - with this being an internal scale specific to each rat. Behaviors proximal to the cat could be related to panic (and were sensitive to panicolytic drugs), whereas more distal behaviors could be related to phobia. Second, they distinguished categorically between fear-related (cat present) and anxiety-related behaviors (cat potentially present) and discovered that the latter but not the former were sensitive to anxiolytic drugs. 


\section{Graeff 1994 - Matching behavioral with neural "levels"}

For me, the most important advance in this whole theoretical progression was provided by Graeff (1994) in a paper in the Brazilian Journal of Medical and Biological Research. He combined the hierarchical notion of defensive distance with the notion of levels in the brain to produce a coherent mapping scheme between the two with charactertistic behaviors defining each level (Table 2). Some important features of this table are: 1) his treatment of an uncertain predator as being even further away than a distant but certain one, producing a system tied to defensive distance; 2) his mapping of the medial hypothalamus to fear (essentially including Flynn's earlier views), the septo-hippocampal system to anxiety (essentially including Gray's theory), and his mapping of the amygdala to anxiety (agreeing with part of LeDoux's theory); and 3) his mapping of the periaqueductal gray to panic.

However, there were some features of this scheme that appeared to me to be problematic. First, derived from the Blanchards' point of view, is the treatment of anxiety as uncertain. The paradigmatic approachavoidance conflict studied by Miller involves no uncertainty - just a balance between known appetitive and aversive drives. Second, is a departure from the Blanchards' formulation in treating anxiety as simply at a greater defensive distance than fear. They applied the concept of defensive distance to both anxiety and fear - and all anxious behavior, independent of defensive distance, is sensitive to anxiolytic drugs, whereas fear behavior is not.

This led me, via the requirement to provide a sound teleonomic account of fear and anxiety, to the following simple definitions. Fear is all those behaviors, autonomic and other reactions, that have evolved in response to the evolutionary pressure of removing the animal from danger. Thus, freezing, fighting and fleeing all contribute to defensive avoidance. Conversely, and essentially opposite to fear, anxiety is all those behaviors, autonomic and other reactions, that have evolved in response to the evolutionary pressure of allowing the animal to approach danger. Thus, defensive quiescence (which is posturally distinct from freezing), risk assessment and behavioral inhibition all contribute to defensive approach (Blanchard \& Blanchard, 1990; Gray \& McNaughton, 2000).

\section{McNaughton \& Corr 2004 - The 2D defense system}

The fundamentals of an essentially two-dimensional model of defense (combining defensive distance with what can be seen as defensive direction, i.e., defensive approach versus defensive avoidance) were put together by me in a revision (Gray \& McNaughton, 2000) of Gray's 1982 theory. But this model was not symmetrical and data continued to accumulate suggesting that it was incomplete. This, coupled with persistent questioning of the details of the theory in relation to human personality by Corr, led to our joint production of the symmetrical 2D model of defense. This is shown with minor further updating (see legend) in Figure 11.

To some extent this model is straightforward with normal behaviours and clinical conditions assigned to particular modules of a 2D system that combines Miller and Gray's fear/anxiety distinction with Graeff's neural translation of the Blanchards' hierarchical ideas. However, superimposed on these modules is diffuse noradrenergic and serotonergic innervation. The inclusion of these systems on anatomical grounds allows the theory to account (with certain assumptions being made about receptor subtypes) for the behavioural and therapeutic effects of a variety of drugs here. An important feature of the serotonin system as shown in Figure 11 derives from the views of Deakin and Graefff (1991) who see conditioned fear as inhibiting fight/flight reactions and so panic. This is linked to a proposed dual action of 5HT enhancing most defensive reaction (see + signs in Figure 11) but suppressing panic-related reactions in the periaqueductal gray (see sign in Figure 11). Further evidence for this position has accumulated since then (Graeff \& Del-Ben, 2008). This antithetical effect of serotonin on the periaqueductal gray accounts for a range of otherwise anomalous effects of serotonergic drugs and also for phenomena such as relaxation-induced panic (Graeff, 1994).

Table 2. Levels of defense, their neural substrate and related emotions (Graeff 1994)

\begin{tabular}{llll}
\hline Danger & Behavior & Emotion & CNS \\
\hline Uncertain & Risk assessment & Anxiety & AM/SHS \\
Distal & Freezing/escape & Fear & PAG-MR-SHS/MH \\
Proximal & Fight/flight & Rage/panic & PAG \\
\hline
\end{tabular}

CNS, central nervous system; AM, amygdala; PAG, midbrain periaqueductal gray; MR, median raphe nucleus; SHS, septohippocampal system; MH, medial hypothalamus. 


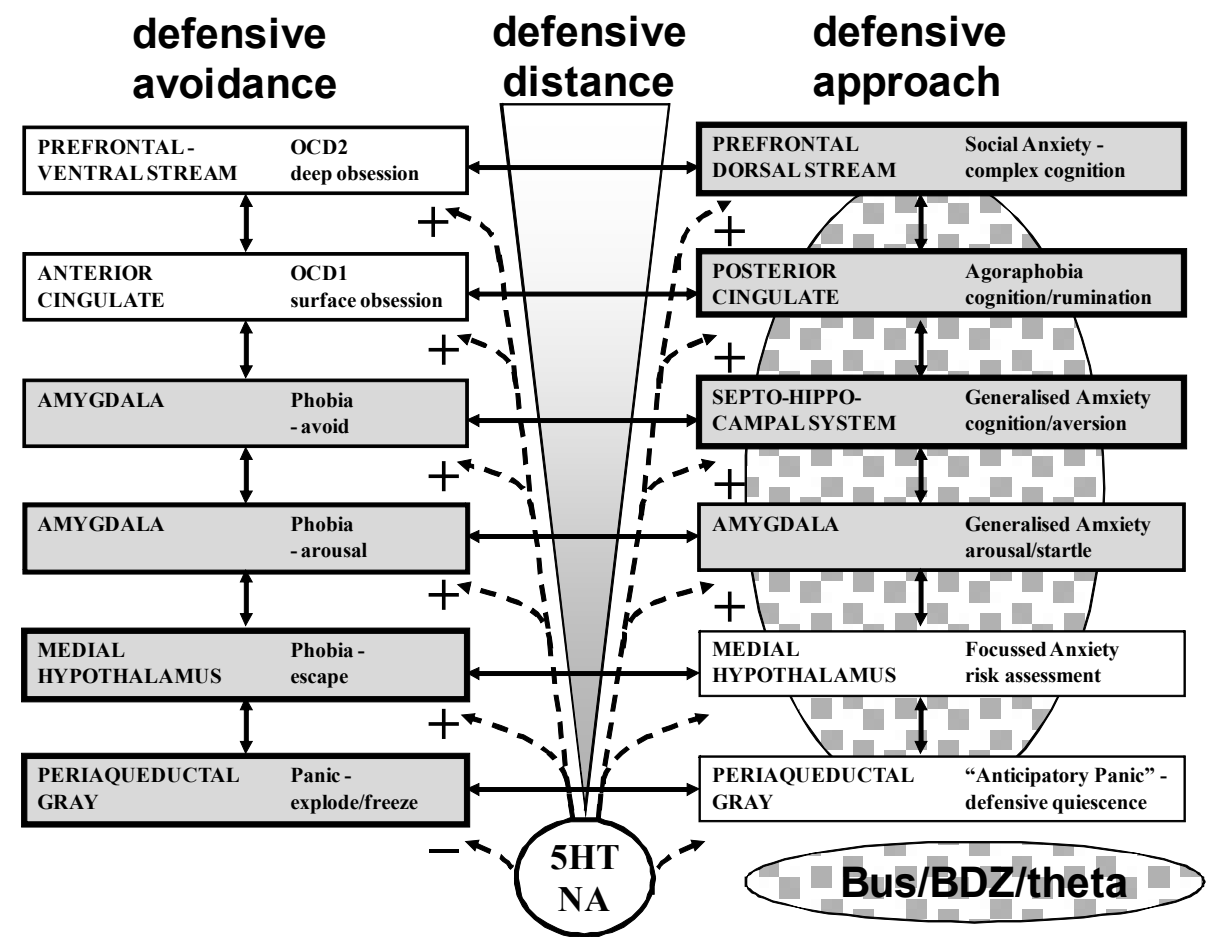

Figure 11. A two-dimensional model of defense systems. There are two systems that differ in defensive direction: defensive avoidance (fear) and defensive approach (anxiety). Both are innervated extensively by serotonin (5HT) and noradrenaline (NA). Buspirone and other 5HT1A agonists (Bus), and benzodiazepines (BDZ) and other classical anxiolytic drugs do not affect the defensive avoidance system and have their strongest effects, mediated by changes in theta rhythm, at the intermediate levels of the defensive approach systems with weaker effects at the lowest and highest levels (stippled area, width indicating extent of action). Normal defensive processes and associated pathologies are allocated to specific levels of each system. Adapted from Gray and McNaughton (2000); McNaughton and Corr (2004).

\section{Future developments}

If this paper were simply a history, it should stop at this point. However, the interest of history is whether we can learn from it. Also, I hope it is clear that this particular history is one of progressive questioning and integration of the available data, producing theories that become progressively more complete, with later theories incorporating earlier ones rather than contradicting them. The development of the "2D model" is clearly still incomplete in a number of major respects so, as I said at the start, I hope this paper can be the kind of starting point for some young researcher that Miller, Flynn and Gray provided for me as an undergraduate. Issues that currently require obvious attention are as follows:

- Neuroeconomics: what is the relation of the 2D theory to the theories of loss aversion, ambiguity aversion and risk aversion? There are good theoretical reasons for seeing loss as orthogonal to approach and avoidance (for example, avoiding loss can produce approach).

- Uncertainty: what is the role of uncertainty of the type emphasised by the Blanchards? It is clearly not the basis for distinguishing anxiety from fear. However, uncertainty is often a factor in generating anxiety - does this have some direct relation to, for example, ambiguity aversion?
-Worry/rumination: this is a clear feature of obsessionality but the current model suggests that there may be both fearful and anxious forms of obsessionality. Further, there are clear data showing that although worry is a risk factor for anxiety disorder, worry and generalised anxiety (once they are present) are not strongly related (Meyer, Miller, Metzger, \& Borkovec, 1990).

- Personality/neuroticism: Neuroticism is a risk factor for both phobic and anxious disorders (and also depression). That is, it predisposes both to disorders that are sensitive to anxiolytics and those that are not. Does this involve some system such as the monoamine ones that provide diffuse input to the entire defensive system?

- Frontal cortex: It is important to note that the inclusion of prefrontal cortex as a single box in Figure 11 ignores the fact that it actually encompasses a substantial proportion of the brain. Disentangling the contribution of all its different areas can potentially answer many or all of the previous bullet point questions.

- Stress: the hippocampus is a key structure within the anxiety control network. How is this related to the fact that the hippocampus has one of the highest concentrations of corticosterone receptors in the brain and is a key structure for the negative feedback control 
of corticosterone and is involved in post-traumatic stress disorder and depression?

There are clearly many more questions to be asked. I hope this paper will help you frame the questions and find their answers.

\section{Acknowledgments}

To the late Professor Jeffrey Alan Gray who was my undergraduate teacher, unofficial advisor to my $\mathrm{PhD}$ and collaborator and co-author for more than 30 years. To my postgraduate students who did the real work and tried to keep me out of mischief: Ann Monahan, Barry Milne, Calvin Young, Colleen Coop, Damon Mitchell, Elizabeth Money, Estella Quintero, Gail Tripp, Gemma Irvine, Ian Kirk, Kiran Panickar, Mary-Anne Woodnorth, Ming Ruan, Nicola Swain-Campbell, Nikki Almond, Phoebe Neo, Rachel Kyd, Rob Munn, Susan Cowie, WeiXing Pan, Xiao-Ou Zhu. And to Philip Corr, whose persistent questioning led to the full 2D model and who is teaching me about personality.

\section{References}

Blanchard, R. J., \& Blanchard, D. C. (1990). An ethoexperimental analysis of defense, fear and anxiety. In N. McNaughton \& G. Andrews (Eds.), Anxiety (pp. 124-133). Dunedin: Otago University Press.

Coop, C. F., \& McNaughton, N. (1991). Buspirone affects hippocampal rhythmical slow activity through serotonin1A rather than dopamine D2 receptors. Neuroscience, 40, 169-174.

Coop, C. F., McNaughton, N., \& Lambie, I. (1991). Effects of GABAA and GABAB receptor agonists on reticular-elicited hippocampal rhythmical slow activity. European Journal of Pharmacology, 192, 103-108.

Coop, C. F., McNaughton, N., \& Scott, D. J. (1992). Pindolol antagonizes the effects on hippocampal rhythmical slow activity of clonidine, baclofen and 8-OH-DPAT, but not chlordiazepoxide and sodium amylobarbitone. Neuroscience, 46, 83-90.

Coop, C. F., McNaughton, N., Warnock, K., \& Laverty, R. (1990). Effects of ethanol and Ro 15-4513 in an electrophysiological model of anxiolytic action. Neuroscience, 35, 669-674.

Deakin, J. F. W., \& Graeff, F. G. (1991). 5-HT and mechanisms of defence. Journal of Psychopharmacology, 5(4), 305-315.

Flynn, J. P. (1967). The neural basis of aggression in cats. In D. C. Glass (Ed.), Neurophysiology and Emotion (pp. 40-60). New York: Rockefeller University Press.

Graeff, F. G. (1994). Neuroanatomy and neurotransmitter regulation of defensive behaviors and related emotions in mammals. Brazilian Journal of Medical and Biological Research, 27, 811-829.

Graeff, F. G., \& Del-Ben, C. M. (2008). Neurobiology of panic disorder: From animal models to brain neuroimaging. Neuroscience and Biobehavioral Reveiws, 32, 1326-1335.

Gray, J. A. (1967). Disappointment and drugs in the rat. Advancement of Science, 23, 595-605.

Gray, J. A. (1970). Sodium amobarbital, the hippocampal theta rhythm and the partial reinforcement extinction effect. Psychological Review, 77, 465-480.

Gray, J. A. (1971). The psychology of fear and stress. London: Weidenfeld and Nicolson.

Gray, J. A. (1977). Drug effects on fear and frustration: possible limbic site of action of minor tranquilizers. In L. L. Iversen, S. D. Iversen, \& S. H. Snyder (Eds.), Handbook of psychopharmacology. Vol 8. Drugs, neurotransmitters and behaviour (pp. 433-529). New York: Plenum Press.

Gray, J. A. (1982). The Neuropsychology of Anxiety: An enquiry in to the functions of the septo-hippocampal system. Oxford: Oxford University Press.

Gray, J. A., \& Ball, G. G. (1970). Frequency-specific relation between hippocampal theta rhythm, behavior and amobarbital action. Science, 168, 1246-1248.

Gray, J. A., \& McNaughton, N. (1983). Comparison between the behavioural effect of septal and hippocampal lesions: a review. Neuroscience and Biobehavioral Reviews, 7, 119-188.

Gray, J. A., \& McNaughton, N. (2000). The Neuropsychology of Anxiety: An enquiry into the functions of the septo-hippocampal system. Oxford: Oxford University Press.

Hinde, R. A. (1966). Animal behaviour. New York: McGraw-Hill Book Company.

James, W. (1884). What is an emotion? Mind, 9, 188-205.

LeDoux, J. E. (1994). Emotion, memory and the brain. Scientific American, 270, 50-59.

McNaughton, N. (1977). Exploration, frustration and the electrophysiology of the septohippocampal theta system in the rat. $\mathrm{PhD}$, Southampton University.

McNaughton, N. (1989). Biology and Emotion. Cambridge: Cambridge University Press.

McNaughton, N., \& Coop, C. F. (1991). Neurochemically dissimilar anxiolytic drugs have common effects on hippocampal rhythmic slow activity. Neuropharmacology, 30, 855-863.

McNaughton, N., \& Corr, P. J. (2004). A two-dimensional neuropsychology of defense: fear/anxiety and defensive distance. Neuroscience and Biobehavioral Reviews, 28, 285-305.

McNaughton, N., Kocsis, B., \& Hajós, M. (2007). Elicited hippocampal theta rhythm: a screen for anxiolytic and pro-cognitive drugs through changes in hippocampal function? Behavioural Pharmacology, 18, 329-346.

McNaughton, N., \& Mason, S. T. (1980). The neuropsychology and neuropharmacology of the dorsal ascending noradrenergic bundle a review. Progress in Neurobiology, 14, 157-219.

McNaughton, N., \& Sedgwick, E. M. (1978). Reticular stimulation and hippocampal theta rhythm in rats: effects of drugs. Neuroscience, 3, 629-632.

Meyer, T. J., Miller, M. L., Metzger, R. L., \& Borkovec, T. D. (1990). Development and validation of the Penn state worry questionnaire. Behaviour Research and Therapy, 28, 487-495.

Miller, N. E. (1944). Experimental studies of conflict. In J. M. Hunt (Ed.), Personality and the behavior disorders (pp. 431-465). New York: The Ronald Press Company.

Munn, R. G. K., \& McNaughton, N. (2008). Effects of fluoxetine on hippocampal rythmic slow activity and behavioural inhibition. Behavioural Pharmacology, 19, 257-264.

Pittendrigh, C. S. (1958). Adaptation, natural selection and behaviour In A. Roes \& G. G. Simpson (Eds.), Behaviour and Evolution (Vol. 1). New Haven: Yale University Press.

Rapoport, J. L. (1989). The biology of obsessions and compulsions Scientific American, 260, 63-69.

Vanderwolf, C. H. (1969). Hippocampal electrical activity and voluntary movement in the rat. Electroencephalography and Clinical Neurophysiology, 26, 407-418.

Zhu, X. O., \& McNaughton, N. (1991a). Effects of long-term administration of anxiolytics on reticular-elicited hippocampal rhythmical slow activity. Neuropharmacology, 30, 1095-1099.

Zhu, X. O., \& McNaughton, N. (1991b). Effects of long-term administration of imipramine on reticular-elicited hippocampal rhythmical slow activity. Psychopharmacology, 105, 433-438.

Zhu, X. O., \& McNaughton, N. (1994). The interaction of serotonin depletion with anxiolytics and antidepressants on reticular-elicited hippocampal RSA. Neuropharmacology, 33, 1597-1605.

Zhu, X. O., \& McNaughton, N. (1995). Effects of long-term administration of phenelzine on reticular- elicited hippocampal rhythmical slow activity. Neuroscience Research, 21, 311-316. 